\title{
Probabilistic vortex crossing criterion for superconducting nanowire single-photon detectors
}

Cite as: J. Appl. Phys. 127, 143101 (2020); https://doi.org/10.1063/1.5132961

Submitted: 22 October 2019 . Accepted: 21 March 2020 . Published Online: 09 April 2020

Saman Jahani (D), Li-Ping Yang (D), Adrián Buganza Tepole, Joseph C. Bardin (D), Hong X. Tang (D), and Zubin Jacob (iD)

ARTICLES YOU MAY BE INTERESTED IN

Dissipative couplings in cavity magnonics

Journal of Applied Physics 127, 130901 (2020); https://doi.org/10.1063/1.5144202

Engineering lattice metamaterials for extreme property, programmability, and multifunctionality

Journal of Applied Physics 127, 150901 (2020); https://doi.org/10.1063/5.0004724

Spin-transport in superconductors

Applied Physics Letters 116, 130501 (2020); https://doi.org/10.1063/1.5138905

\section{Lock-in Amplifiers up to $600 \mathrm{MHz}$}
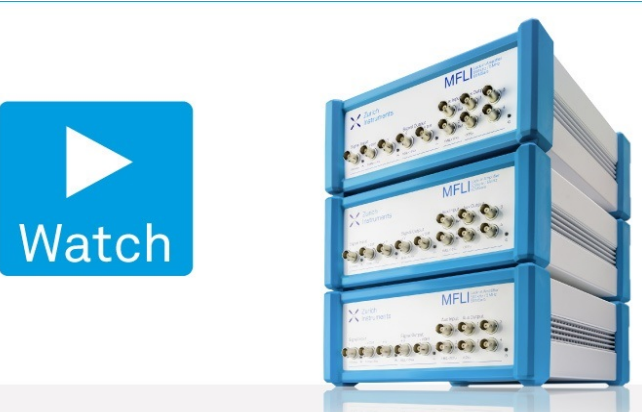


\title{
Probabilistic vortex crossing criterion for superconducting nanowire single-photon detectors
}

\author{
Cite as: J. Appl. Phys. 127, 143101 (2020); doi: 10.1063/1.5132961 \\ Submitted: 22 October 2019 - Accepted: 21 March 2020 . \\ Published Online: 9 April 2020
}

Saman Jahani, , ,a) (iD Li-Ping Yang, ${ }^{1}$ (D) Adrián Buganza Tepole, ${ }^{2}$ Joseph C. Bardin, ${ }^{3}$ (iD Hong X. Tang, ${ }^{4}$ and Zubin Jacob ${ }^{1, b)}$ (iD

\author{
AFFILIATIONS \\ ${ }^{1}$ School of Electrical and Computer Engineering and Birck Nanotechnology Center, Purdue University, West Lafayette, \\ Indiana 47907, USA \\ ${ }^{2}$ School of Mechanical Engineering, Purdue University, West Lafayette, Indiana 47907, USA \\ ${ }^{3}$ Department of Electrical and Computer Engineering, University of Massachusetts at Amherst, Amherst, Massachusetts 01003, \\ USA \\ ${ }^{4}$ Department of Electrical Engineering, Yale University, New Haven, Connecticut 06511, USA
}

a)Current address: Moore Laboratory, Department of Electrical Engineering, California Institute of Technology, Pasadena, CA 91125, USA.

${ }^{\text {b) Author to whom correspondence should be addressed: } z j a c o b @ p u r d u e . e d u}$

\begin{abstract}
Superconducting nanowire single-photon detectors have emerged as a promising technology for quantum metrology from the mid-infrared to ultraviolet frequencies. Despite recent experimental successes, a predictive model to describe the detection event in these detectors is needed to optimize the detection metrics. Here, we propose a probabilistic criterion for single-photon detection based on single-vortex (flux quanta) crossing the width of the nanowire. Our model makes a connection between the dark counts and photon counts near the detection threshold. The finite-difference calculations demonstrate that a change in the bias current distribution as a result of the photon absorption significantly increases the probability of single-vortex crossing even if the vortex potential barrier has not vanished completely. We estimate the instrument response function and show that the timing uncertainty of this vortex tunneling process corresponds to a fundamental limit in timing jitter of the click event. We demonstrate a trade-space between this intrinsic (quantum) timing jitter, quantum efficiency, and dark count rate in $\mathrm{TaN}, \mathrm{WSi}$, and $\mathrm{NbN}$ superconducting nanowires at different experimental conditions. Our detection model can also explain the experimental observation of exponential decrease in the quantum efficiency of SNSPDs at lower energies. This leads to a pulse-width dependency in the quantum efficiency, and it can be further used as an experimental test to compare across different detection models.
\end{abstract}

Published under license by AIP Publishing. https://doi.org/10.1063/1.5132961

\section{INTRODUCTION}

Advancements in quantum technologies strongly depend on the improvement in the detection of light at the single-photon level. This requires near-unity quantum efficiency, sub-picosecond timing uncertainty (timing jitter), sub-milihertz dark count rate, large bandwidth, and fast reset time. ${ }^{1}$ Superconducting nanowire single photon detectors (SSPDs or SNSPDs) are highly promising detectors in a broad range of frequencies from mid-infrared to ultraviolet $^{2-7}$ with near-unity quantum efficiency, ${ }^{8}$ picosecond-scale timing jitter, $^{9-12}$ fast reset time, ${ }^{13}$ and millihertz dark count rate. $^{14,15}$ They are composed of a thin superconducting nanowire, which is biased slightly below the superconducting critical current. Photon absorption triggers a phase transition giving rise to the generation of a voltage pulse, which is measured by a readout circuit connected to the nanowire. 
Owing to the experimental progress on reducing amplification noise and the uncertainty of the photon absorption location, recent breakthrough results have shown timing jitter below 10 ps. ${ }^{9-11,16,17}$ Hence, the response function of SNSPDs to a single photon has approached its intrinsic response limit, which only depends on the microscopic mechanism of light-matter interaction in nanowires. To further improve the performance of these detectors, it is necessary to understand the microscopic mechanism and the trade-space of the photon detection event in these detectors.

Over the past two decades, several important detection models have been proposed to explain the microscopic mechanism of the formation of the first resistive region in SNSPDs. ${ }^{2,3,18-23}$ In the simplest model, it is assumed that the energy of the absorbed photon increases the temperature at the absorption site, leading to the nucleation of a hot-spot, which causes the current to be directed toward the sides. $^{2,24,25}$ This may cause the current at the edge to surpass the critical depairing current, causing formation of a normal conducting region across the width. In another model, the depletion of superconducting electrons around the absorption site is responsible for the formation of the resistive region. ${ }^{26}$ Recently, some models have suggested that the motion of vortices or vortex-antivortex pairs can also induce a phase transition at a lower applied bias current. ${ }^{18,21,23,27-29}$ Although each of these models explains most of the macroscopic behaviors of SNSPDs, existing models cannot explain or predict the trade-off between the quantum efficiency, timing jitter, and dark counts and their fundamental limits in SNSPDs.

In this paper, we construct a connection between the photon-induced counts and the dark counts in SNSPDs, which has been recently observed experimentally around the detection current. $^{30,31}$ We propose a probabilistic criterion for single-photon detection corresponding to the single-vortex crossing from one edge of the nanowire to the other edge. First, we numerically calculate the time-dependent current distribution after the photon absorption and its effect on the vortex potential. We propose that, due to the change in the distribution of the superconducting electrons, the probability of the vortex crossing is significantly enhanced even if the vortex potential barrier has not vanished completely.

Then, we define the detection probability based on the probability of the single-vortex crossing because the energy released by one vortex moving across the width is enough to induce a phase transition in the superconducting nanowire. We show that the probabilistic behavior of the single-vortex crossing results in an intrinsic timing jitter on the click event. This intrinsic quantum timing jitter cannot be eliminated, even if the geometric position of photon absorption is known; however, it can be reduced by engineering the structure and the experimental conditions at the cost of degradation of the quantum efficiency and/or an increase in the dark count rate.

Finally, we calculate the quantum efficiency spectrum and show that the quantum efficiency does not suddenly drop to zero when the photon energy is below a threshold. We propose that the response of the detector to the photon pulse width can be different for the various detection models. Moreover, the quantum efficiency predicted by our model is strongly dependent on the pulse width. This effect has not been predicted by the previous detection models. Our work unifies previously known ideas of a vortex crossing phenomenon with the positive-operator-valued measure (POVM) approach of quantum optics to propose a probabilistic detection criterion for SNSPDs. We propose some observable quantities which can be used to experimentally verify the validity of our probabilistic model. Our model focuses around the detection threshold (quantum efficiency $\approx 1$ ) where photons do not have enough energy to form a normal conducting hot-spot and the probabilistic behavior of vortices is more significant. This is not in contradiction to observations of the vortex/anti-vortex pair unbinding. For higher energies or higher bias currents, the formation of a hot-spot and, as a result, vortex/anti-vortex pair unbinding might happen before the probabilistic tunneling of a single-vortex from the edges. ${ }^{23,32}$

\section{DETECTION MECHANISM}

Detection mechanism in SNSPDs consists of three steps: (a) photon absorption and breaking the superconducting electron pairs (known as Cooper pairs) to quasi-particles (QPs) leading to the formation of a hot-spot; (b) as a result of the depletion of the Cooper pairs, the superconducting order parameter is suppressed. This causes the current density at the absorption location to be reduced and directed to the sides as illustrated in Fig. 1(a); and (c)
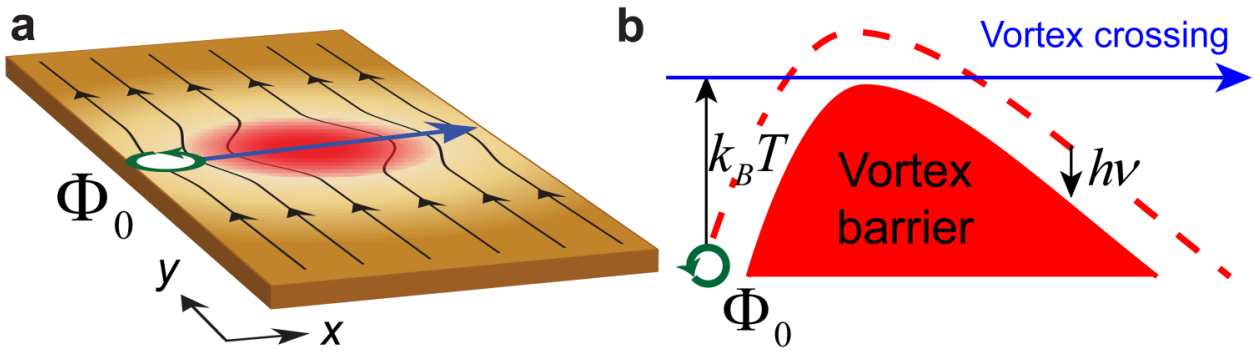

FIG. 1. Superconducting nanowire single-photon detectors (SNSPDs). (a) When a photon falls on the detector, quasi-particles (QPS) are generated and the bias current is redistributed. Vortices with a magnetic flux quantum of $\Phi_{0}$ are the topological defects of a thin superconductor and are nucleated at the nanowire edge. They can move to the other edge due to the force exerted by the bias current. (b) Before the photon absorption, the vortex potential barrier does not allow them to move easily. However, due to the QP multiplication and current redistribution, the barrier reduces and vortices, which are thermally excited, can escape the barrier and cross the width of the nanowire. This process generates a voltage pulse propagating to the two ends of the detector. We provide a probabilistic click definition using this detection event. 
the change in the Cooper pairs and current density reduces the vortex potential barrier, and vortices can move across the nanowire and release a measurable voltage pulse [Fig. 1(b)].

These three steps have been quantitatively described in the Appendix. Our finite-difference calculations of QP distribution based on the diffusion model ${ }^{21}$ for a TaN SNSPD are illustrated in Figs. 2(a) and 2(b) at $t=1 \mathrm{ps}$ and $t=5 \mathrm{ps}$, respectively. We assume a photon with the energy of $h v=1.5 \mathrm{eV}$ falls at the center of the SNSPD at $t=0$. The width and the length of the nanowire
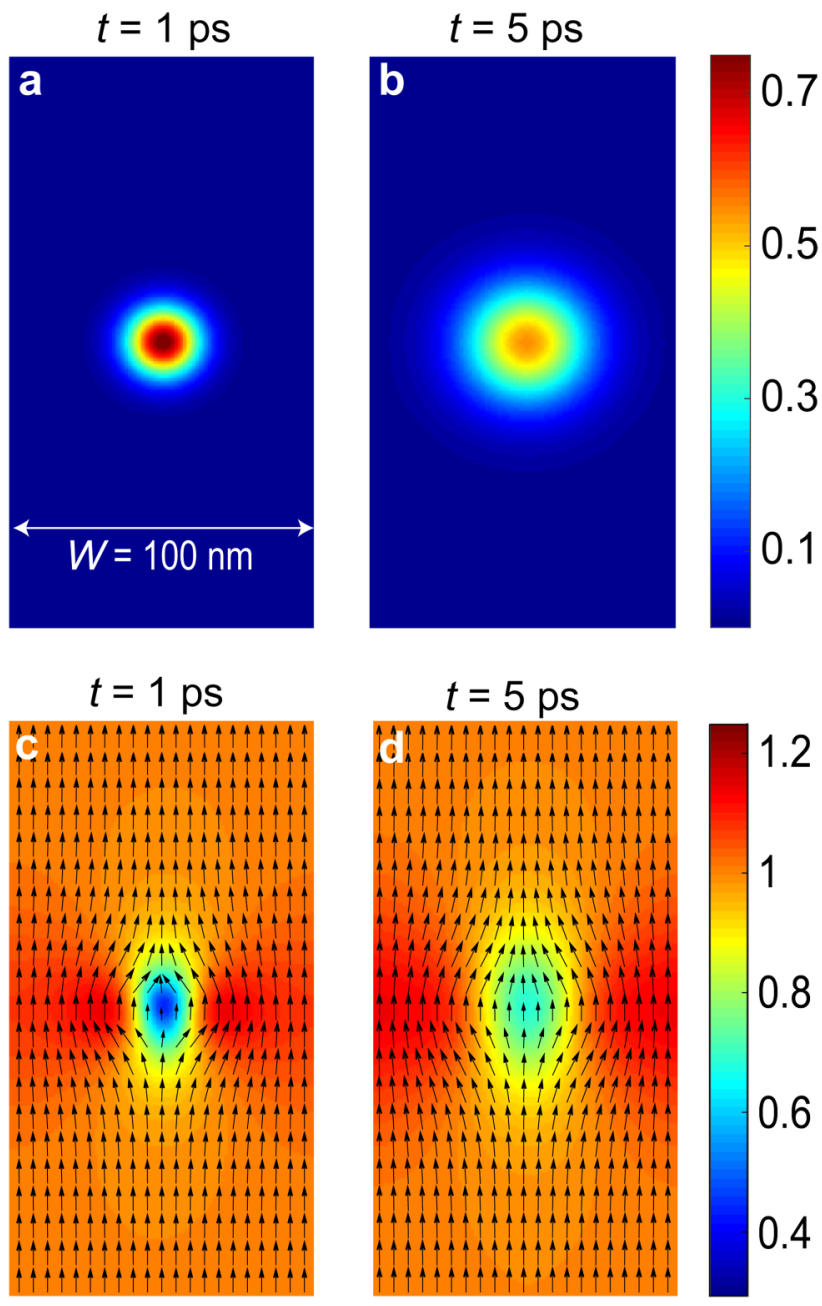

FIG. 2. (a) and (b) QP distribution, $C_{q p}(\vec{r}, t)$, normalized to the initial density of superconducting electrons, $n_{s e, 0}$, in a TaN SNSPD at $t=1 \mathrm{ps}$ and $t=5 \mathrm{ps}$, respectively. It is assumed that the photon is absorbed at $t=0 . T=0.6 \mathrm{~K}$. The photon energy is $h v=1.5 \mathrm{eV}$. The nanowire width, length, and thickness are $100 \mathrm{~nm}, 1000 \mathrm{~nm}$, and $5 \mathrm{~nm}$, respectively. (c) and (d) Normalized current density in the $y$ direction at $t=1 \mathrm{ps}$ and $t=5 \mathrm{ps}$, respectively. The current density is normalized to the applied bias current. The arrows represent the current density vector, $\vec{j}(\vec{r}, t)$. Due to the hot-spot formation, current is redistributed and directed to the sidewalls. are $W=100 \mathrm{~nm}$ and $L=1000 \mathrm{~nm}$, respectively. Figures 2(c) and 2(d) display the numerical calculation of the current density normalized to the bias current. It is seen that due to the hot-spot formation at the center, the current is directed to the sidewalls of the nanowire, and as a result, the vortex potential barrier is reduced as shown in Fig. 3. If the bias current or the photon energy are high enough, the potential barrier can be vanished completely.

After the single-photon transduction, several processes compete with each other to form the initial normal conducting cross section. Depending on which one occurs first, different detection models have been proposed. In the hot-spot model, it is assumed that the formation of the hot-spot is responsible for the phase transition. ${ }^{2,25}$ Nucleation of the hot-spot causes the bias current to be directed to the sidewalls. If the current density at the
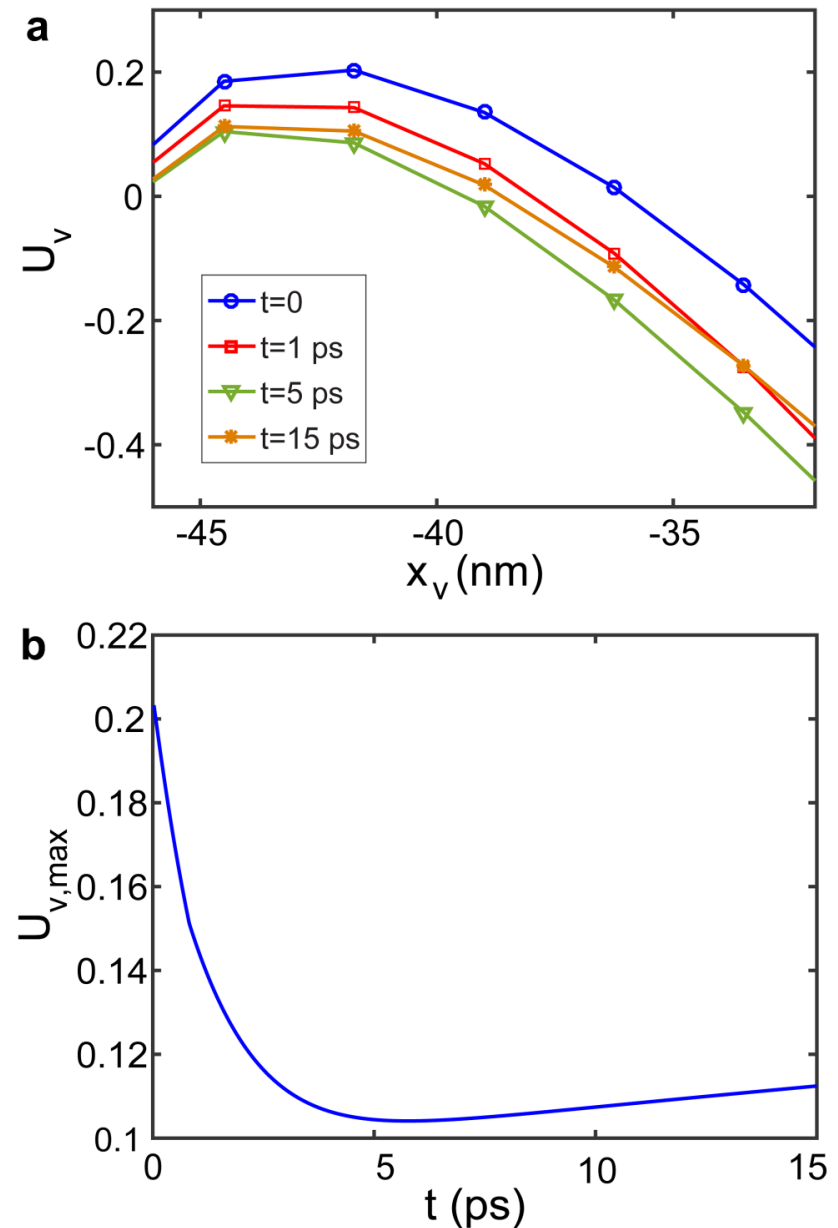

FIG. 3. Vortex barrier dynamics. (a) Vortex potential as a function of the vortex location, $x_{v}$, around the saddle point after the photon absorption for a TaN SNSPD. $T=0.6 \mathrm{~K}, W=100 \mathrm{~nm}$, and $h v=1.5 \mathrm{eV}$. (b) Potential barrier peak as a function of time. A change in the Cooper pairs density and current distribution reduce the barrier height. The potential has been normalized to the characteristic vortex energy, $\varepsilon_{0}$ 
edge surpasses the despairing critical current $\left(I_{\text {edge }} \geq I_{c, \text { dep }}\right)$, it induces a phase transition to the normal conducting state at the edge, and the normal conducting region expands across the width.

In the QP model, there is no need to destroy the superconductivity by surpassing the critical current. ${ }^{26}$ If the Cooper pairs are depleted inside a volume with a thickness of at least one coherence length $(\xi$-slab), the phase coherence is destroyed, which results in a phase transition. This requires the number of QPs inside the $\xi$-slab $\left(N_{Q P}^{s l a b}\right)$ to exceed the number of the superconducting electrons inside the $\xi$-slab: $N_{Q P}^{\text {slab }} / N_{s e}^{\text {slab }} \geq 1-I_{b} / I_{c, \text { dep }}$, where $N_{s e}^{\text {slab }}$ is the initial superconducting-electron number inside the slab before applying the bias current $\left(I_{b}\right)$.

Vortices can also be responsible for the trigger of a single-photon-induced phase transition in SNSPDs. If the photon transduction causes the vortex potential barrier $\left(U_{v}\right)$ to vanish, vortices move across the width and induce a phase transition. ${ }^{20,21}$ In Sec. III, we show that even if the barrier has not completely vanished and the kinetic energy of the vortices is not enough to surmount the barrier, there is a considerable probability of single-vortex crossing. This quantum tunneling process causes a new source of timing jitter for the detection event.

\section{QUANTUM TIMING JITTER}

According to the most accepted quantum measurement theory, positive-operator-valued measure (POVM), a quantum detector can be regarded as a black box. Each of its outcomes is represented by a positive Hermitian operator $\hat{\Pi}_{m}$ with non-negative real eigenvalues. The probability that the $m$ th outcome occurs in experiments is given by $P_{m}=\operatorname{Tr}\left[\rho \hat{\Pi}_{m}\right]$, where $\rho$ is the initial state of the quantum object to be detected, such as the state of the incident single-photon pulse. The completeness condition, $\sum_{m} \hat{\Pi}_{m}=\hat{I}$ ( $\hat{I}$ is the identity operator), expresses the fact that the probabilities sum to one: $\sum_{m} P_{m}=1$. For a non-photon-number-resolving photon detector, there are only two possible outcomes: clicking and non-clicking, characterized by $\hat{\Pi}_{c}$ and $\hat{\Pi}_{n c}$ (thus, $m=c, n c$ ), respectively. The clicking probability, $P_{c}=\operatorname{Tr}\left[\rho \hat{\Pi}_{c}\right] \equiv P_{1}+P_{0}$, contains two parts: the single-photoninduced clicking probability, $P_{1}$, characterizing the quantum efficiency of the detector and the dark counting part, $P_{0}$. Note that we have neglected the nonlinearity in the detector response. ${ }^{34,35}$ Recently, the figures of merit and time-dependent spectrum of a single photon in terms of POVMs have been exploited. ${ }^{36,37}$ In the following, we present our microscopic calculation of $P_{1}$ and $P_{0}$ based on the single-vortex crossing model. Particularly, we introduce the quantum timing jitter in the amplification process, which has not been incorporated into the current POVM theory.

Even if there is no photon and the bias current is below the vortex critical current $I_{c, v}$, a vortex can be thermally excited and escape the potential barrier saddle point to form a normal conducting belt. ${ }^{38,39}$ This false-count rate is known as a dark-count rate, which deteriorates the performance of a single-photon detector. ${ }^{1}$ The time-dependent rate of the vortex crossing can be described as

$$
\Gamma_{v}(t)=\alpha_{v} I_{b} \exp \left(-U_{v, \max }(t) / k_{B} T\right),
$$

where $k_{B}$ is the Boltzmann constant and $\alpha_{v}$ is a constant, which is measured experimentally. ${ }^{38} U_{v, \max }(t)$ is the maximum of the potential barrier for vortex crossing, which changes with time after the single-photon absorption event. As a result of the change in the vortex potential barrier after the photon absorption, the vortex crossing rate increases exponentially. Figure 4(a) shows the vortex crossing rate as a function of time after the photon absorption for three different materials. The rate at $t=0$ corresponds to the darkcount rate. ${ }^{38,40-42}$ However, the rate is enhanced several orders of
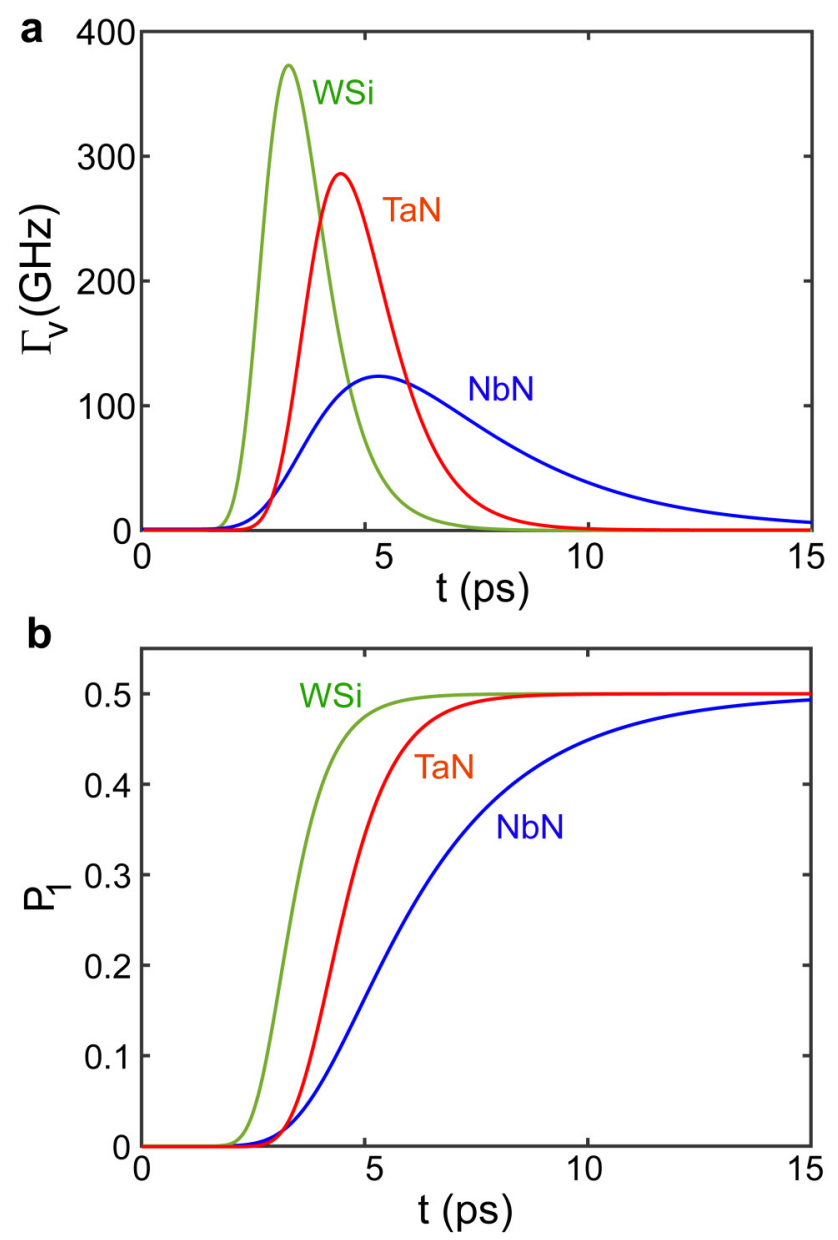

FIG. 4. Vortex crossing rate and probability. (a) Single-vortex crossing rate as a function of time for NbN, TaN, and WSi SNSPDs. The bias current has been set to achieve a single-photon detection probability of 0.5 for a photon energy of $h v=1.5 \mathrm{eV}$, which is $0.96 / \mathrm{sw}, 0.93 / \mathrm{sw}$, and $0.65 / \mathrm{sw}$, for NbN, TaN, and WSi SNSPDs, respectively. The reduced temperatures $\left(T / T_{c}\right)$ for $\mathrm{TaN}, \mathrm{NbN}$, and WSi at $T=0.6 \mathrm{~K}$ are $0.07,0.05$, and $0.15 . W=100 \mathrm{~nm}$. $I_{\mathrm{sW}}$ is defined as the minimum bias current at which the detector clicks in the time bin of the singlephoton arrival even if the photon is not absorbed. Material parameters are derived from Ref. 33. The enhancement in the vortex crossing rate is as a result of the suppression of the potential barrier. The probability of vortex crossing at the maximum rate is higher. However, there is considerable uncertainty in the vortex crossing time, which results in a timing jitter in a detection event. (b) The evolution of vortex crossing probability as the vortex crossing rate changes. There is a steep change in the probability as the crossing rate goes up. 
magnitude when the potential barrier reaches its minimum. This enhancement during the multiplication and recombination of QPs might be enough to significantly change the probability of the vortex escaping the barrier. It is seen that the shape of the vortex crossing rate is different in different superconducting materials depending on the number of QPs generated and how fast they get multiplied, diffused across the width, and recombined. Since the crossing of vortices occurs independent of the other vortices, the crossing events can be regarded as a Poisson process with the distribution function,

$$
p\left(n_{v}, t\right)=\frac{\bar{n}_{v}(t)}{n_{v} !} e^{-\bar{n}_{v}(t)},
$$

characterizing the probability of the $n_{v}$ vortex crossing the nanowire during the time interval $\left[t_{0}, t\right]$. Here, the time-dependent function $\bar{n}_{v}(t)=\int_{t_{0}}^{t} \Gamma_{v}\left(t^{\prime}\right) d t^{\prime}$ is the mean number of vortices crossing the nanowire. Hence, we can define the single-photon detection probability $P_{1}$ after the single-photon absorption $\left(t_{0}=0\right)$ and before time $t$ as the probability of crossing of at least one vortex as

$$
P_{1}(t)=1-p(0, t)=1-\exp \left[-\int_{t_{0}=0}^{t} \Gamma_{v}\left(t^{\prime}\right) d t^{\prime}\right] .
$$

We have neglected the interaction between vortices during the vortex crossing.

As seen in Fig. 4(b), the detection probability increases rapidly around the crossing rate maximum. The time derivative of $P_{1}(t)$ is proportional to the single-photon count rate (also known as an instrument response function) measured in experiments. ${ }^{9}$ If the detection efficiency is low, the photon count rate is approximately the same as $\Gamma_{v}(t)$. The rise time of the quantum efficiency is not instantaneous due to the finite diffusion speed of the QPs and the hot-spot formation. Hence, there is a fundamental latency and uncertainty between the time of photon absorption and the quantum vortex tunneling process. ${ }^{43}$ This causes a quantum timing jitter $\left(t_{j}\right)$ on the photon detection event as illustrated in Fig. 5. As shown in Fig. 6, the latency is lower for higher energy photon detection since the vortex barrier is suppressed faster. The latency is also reduced if the bias current is increased. There is a sharp drop in the latency when the photon detection becomes deterministic.

This type of timing jitter is because of the probabilistic tunneling of vortices, ${ }^{44,45}$ which is nonzero even if the absorption location of the photon is known exactly. In the state-of-the-art experiments, the total jitter is dominated by the geometric jitter as a result of the uncertainty in the position of the transduction event. ${ }^{32,46-51}$ However, even if the geometric jitter is suppressed by defining the exact longitudinal ${ }^{6,52}$ and transverse location of the photon absorption, the quantum jitter cannot be diminished beyond a limit. However, it can be controlled by engineering the structure and controlling the experimental conditions. Note that to make a quantitative comparison between the simulation results and the existing experimental results, the polarization of the incident photon and the absorption location in the transverse co-ordinate must be considered. ${ }^{53}$ This will be undertaken in a future study.

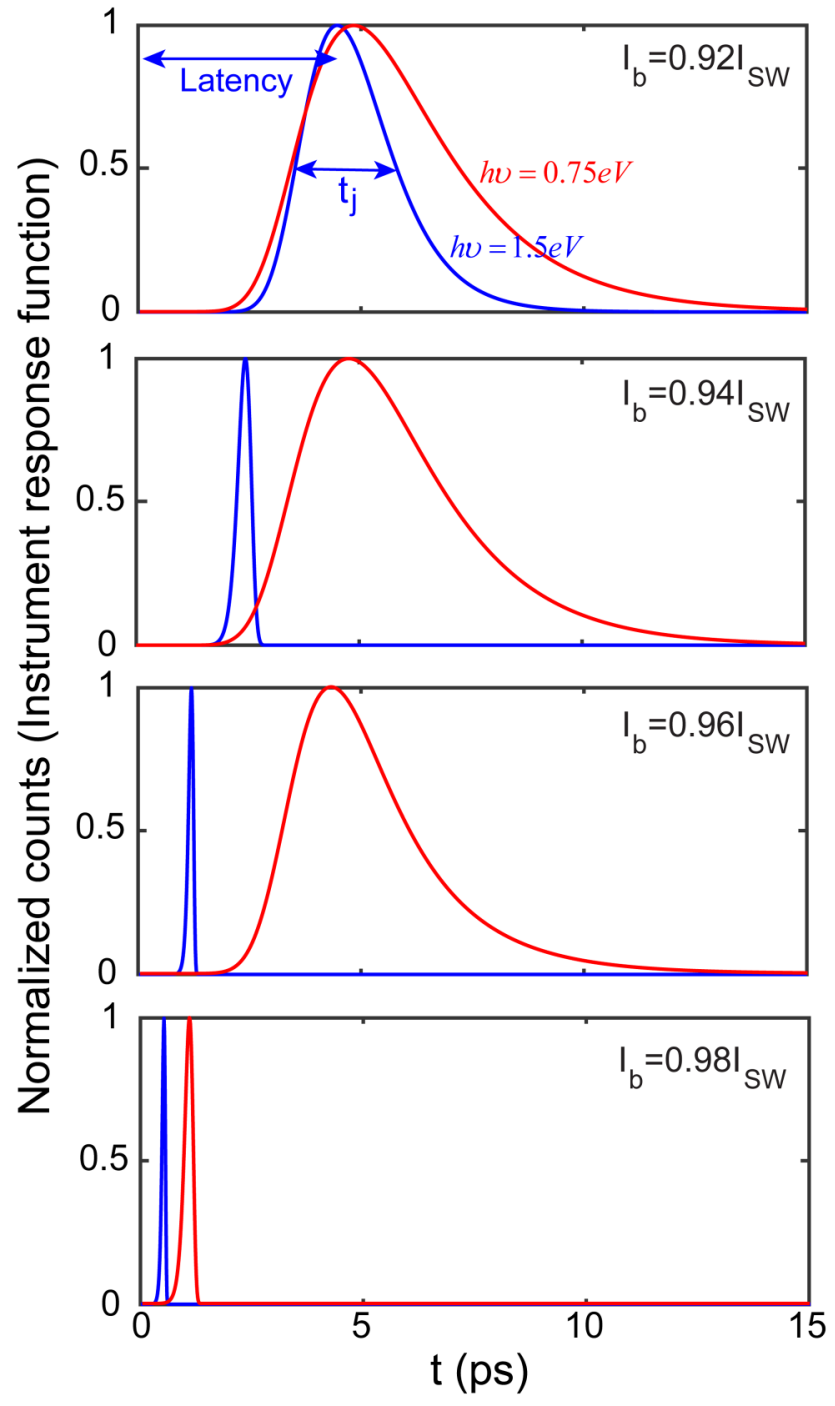

FIG. 5. Instrument response function. Estimated distribution of a number of counts registered on the detector as a function of the delay after the photon absorption in a TaN SNSPD when the incoming single-photon energy is $h v=1.5 \mathrm{eV}$ (blue) and $h v=0.75 \mathrm{eV}$ (red); $T=0.6 \mathrm{~K}$ and $W=100 \mathrm{~nm}$. There is a latency between the photon absorption and the click registration, and the uncertainty in the latency causes a timing jitter $\left(t_{j}\right)$ in the detector.

It is seen in Eq. (1) that as the temperature decreases, the change in the vortex crossing rate becomes sharper. This causes the intrinsic timing jitter to reduce as shown in Fig. 7(a), which is in agreement with recent experiments. ${ }^{54}$ Note that the vortex potential is proportional to the characteristic vortex energy, $\varepsilon_{0}$. Thus, smaller vortex energy causes a slower change in the vortex crossing rate, similar to the effect of a rise in the temperature. Hence, although the hot-spot formation and relaxation happen faster in WSi due to the smaller bandgap and faster QP diffusion, ${ }^{33}$ the timing jitter in 


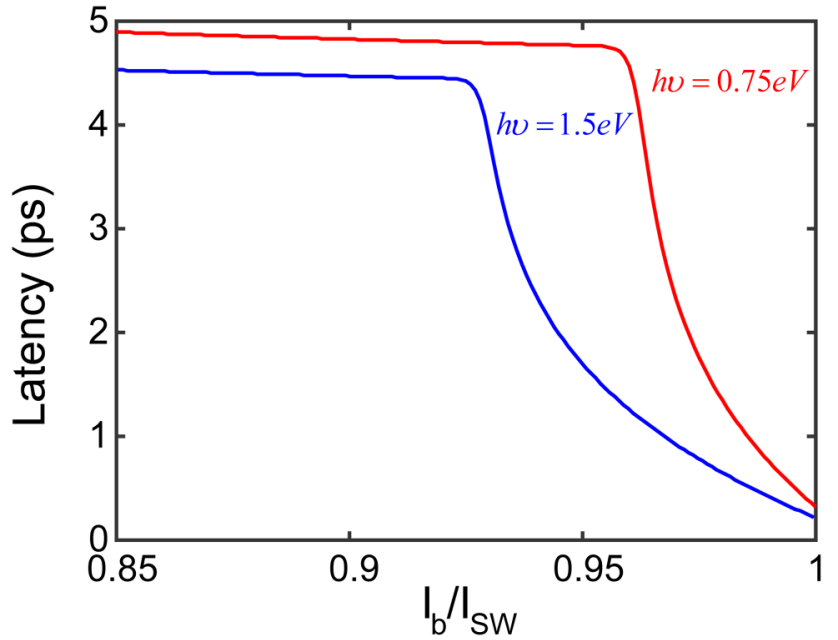

FIG. 6. The effect of bias current on the latency in TaN SNSPDs. The latency suddenly drops when the quantum efficiency approaches unity, and it can be significantly reduced if the bias current becomes very close to the switching current $\left(I_{S W}\right)$ or the photon energy increases. $T=0.6 \mathrm{~K}$ and $W=100 \mathrm{~nm}$.

WSi is comparable with that in TaN because of the smaller $\varepsilon_{0}$ in WSi nanowires.

Reducing the width of the nanowire results in a faster distribution of the hot-spot across the nanowire width. This leads to a sudden change in the vortex potential barrier, and as a result, the timing jitter decreases considerably as shown in Fig. 7(b). Reducing the width helps reducing the geometrical timing jitter as well, ${ }^{46}$ however, at the cost of a decrease in the transduction efficiency of the device.

Increasing the bias current reduces the vortex potential and increases the vortex crossing rate. This causes not only an increase in the quantum efficiency $\left(P_{1}\right),{ }^{5,56}$ but also an increase in the dark count probability $\left(P_{0}\right)$ as shown in Fig. $8 . P_{0}$ is defined as the probability of the click, while there is no interaction between the photon and the detector in the time bin of the photon arrival. $I_{\mathrm{SW}}$ is defined as the minimum bias current, which is required for at least one vortex to escape the barrier in the time bin of the photon arrival. Note that $I_{\mathrm{SW}}$ is lower than the vortex critical current, $I_{c, v}$, especially if the temperature is not low enough. The bias current has also a significant impact on the timing jitter corresponding to single-vortex crossing. If the detector is biased very close to the switching current, a small perturbation due to the single-photon absorption suppresses the potential barrier and vortex can cross the width. Figure 8 displays the effect of the bias current on the timing jitter as well. It is seen that the timing jitter drops remarkably when the quantum efficiency approaches unity in agreement with the recent experimental observations. ${ }^{10,57,58}$ This is because of the significant suppression of the barrier, which leads to the vortex crossing even before the rate reaches its maximum. It is also seen that the slopes of $P_{0}$ and $P_{1}$ are identical in the non-deterministic region in agreement with the recent experiments, ${ }^{30,31}$ which confirms the connection between the photon counts and the dark counts around the detection current.
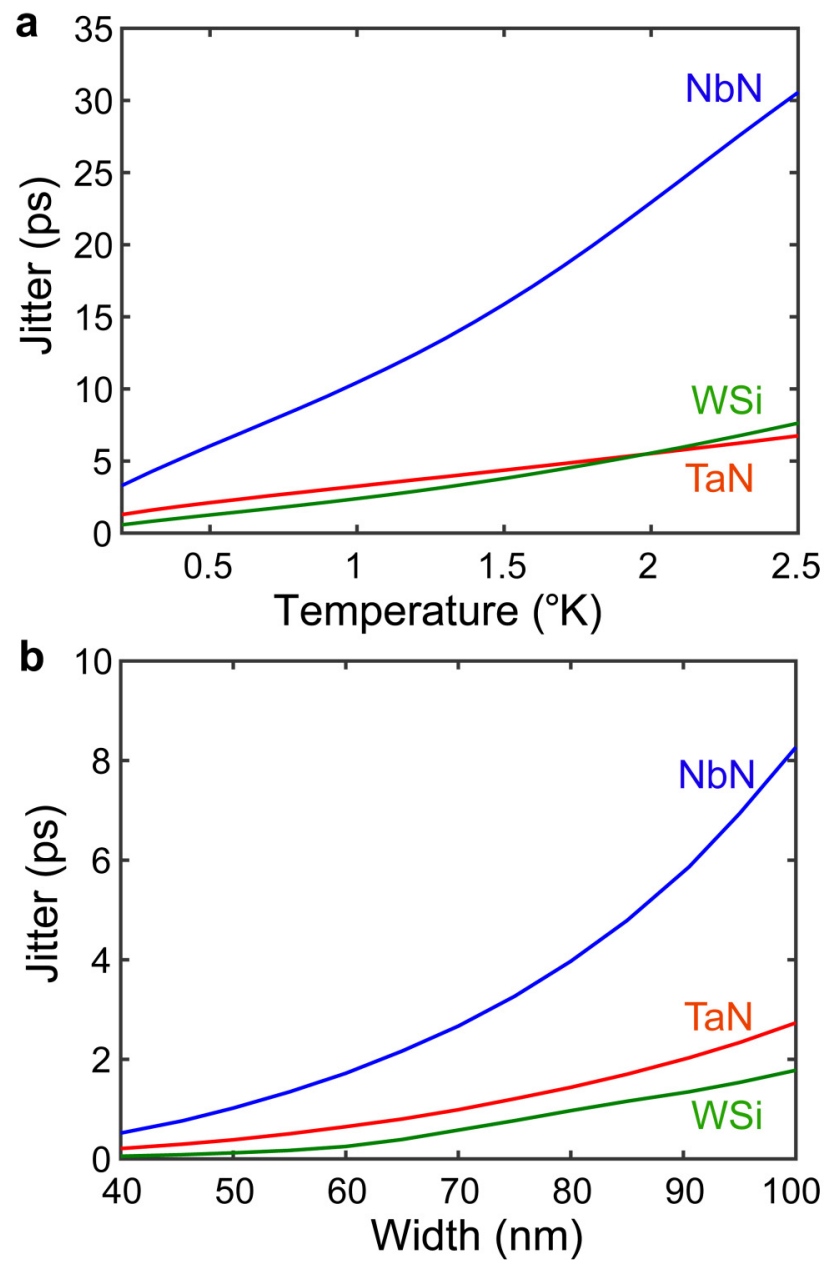

FIG. 7. Timing jitter corresponding to vortex crossing. Timing jitter in $\mathrm{NbN}, \mathrm{TaN}$ and WSi SNSPDs as a function of (a) temperature $(W=100 \mathrm{~nm})$ and $(b)$ nanowire width $(T=0.6 \mathrm{~K}) . h v=1.5 \mathrm{eV}$. Decreasing the temperature results in a sharper change in the vortex crossing rate. Hence, the uncertainty of vortex crossing reduces. Reducing the width of the nanowire causes the QPs to distribute faster across the width of the nanowire, and as a result, the potential barrier reduces rapidly.

\section{SPECTRAL QUANTUM EFFICIENCY}

The non-deterministic behavior of vortices in the case when the potential barrier has not vanished completely allows us to estimate the quantum efficiency probability even for low energy photons. Figure 9 shows the quantum efficiency based on the single-vortex crossing model as a function of the energy of the absorbed single-photon at different temperatures. The bias current is set to have a near-unity quantum efficiency when the photon energy is larger than $1 \mathrm{eV} . \alpha_{v}$ in Eq. (1) can be used as a fitting parameter to define the actual current with respect to the experimental depairing current. The quantum efficiency approaches $P_{0}$ when the photon energy goes to zero. As the photon energy goes 


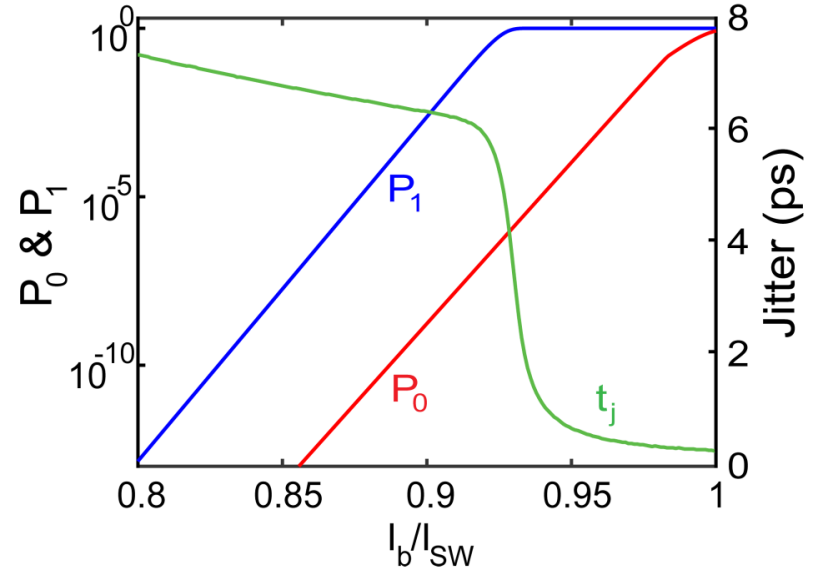

FIG. 8. The effect of bias current on TaN SNSPD performance. Timing jitter, dark count probability $\left(P_{0}\right)$, quantum efficiency $\left(P_{1}\right)$ vs the bias current $\left(I_{b}\right)$. $I_{b}$ is normalized to the switching current. Note that $I_{S w}$ is smaller than $I_{c, v}$. Increasing the bias current helps improving the detection probability and the timing jitter but at the cost of an increase in the dark count probability. $T=0.6 \mathrm{~K}$, $W=100 \mathrm{~nm}$, and $h v=1.5 \mathrm{eV}$.

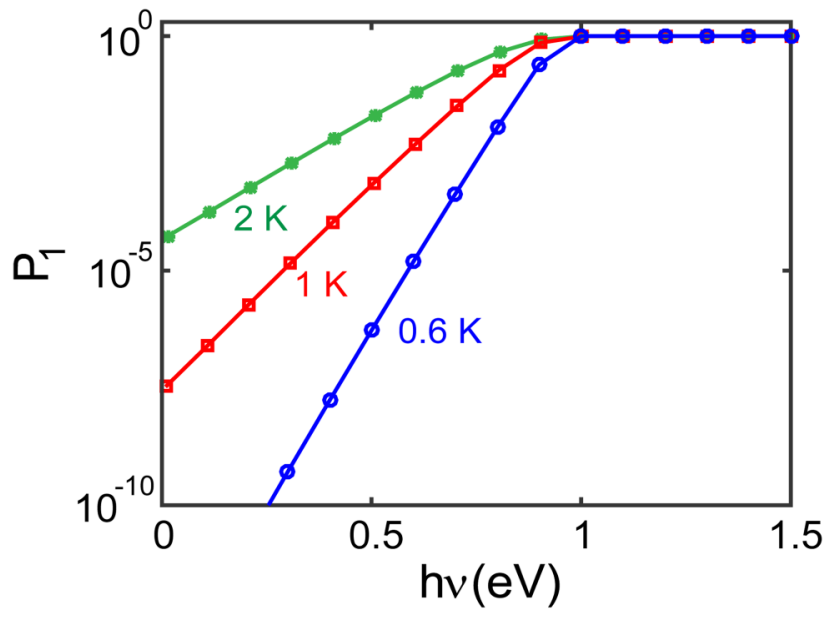

FIG. 9. Single-photon detection probability (quantum efficiency) as a function of single-photon energy and temperature in TaN SNSPDs. The bias current is set to have near-unity detection probability when the photon energy is larger than $1 \mathrm{eV} . W=100 \mathrm{~nm}$. For high energy photons, the vortex potential barrier drops to zero. Hence, the click event, which is as a result of the vortex crossing, happens certainly. However, if the photon energy is not high enough to suppress the potential barrier completely, the vortex crossing event becomes nondeterministic, and it drops exponentially as the photon energy is reduced.
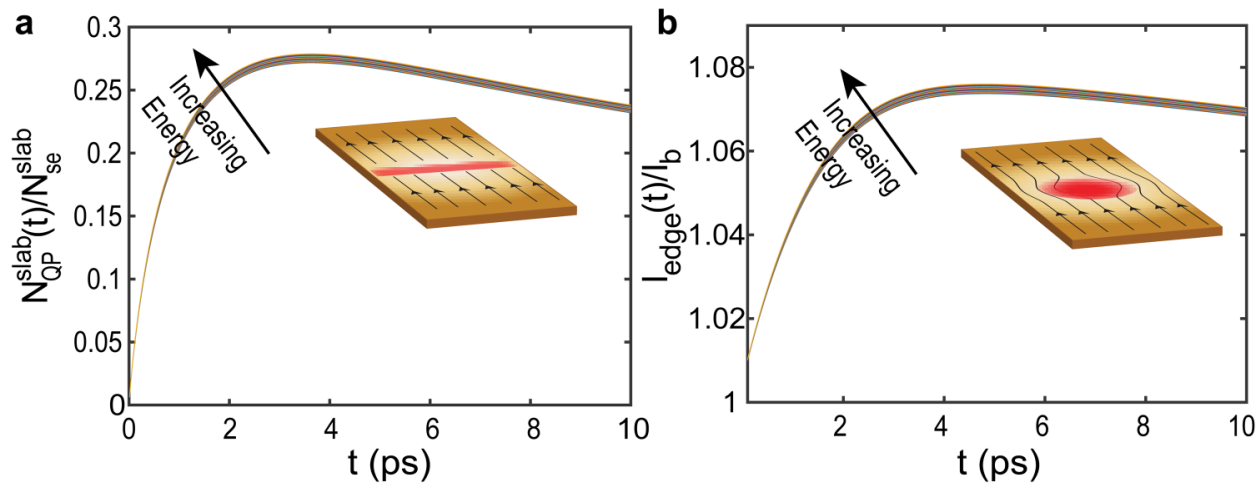

C

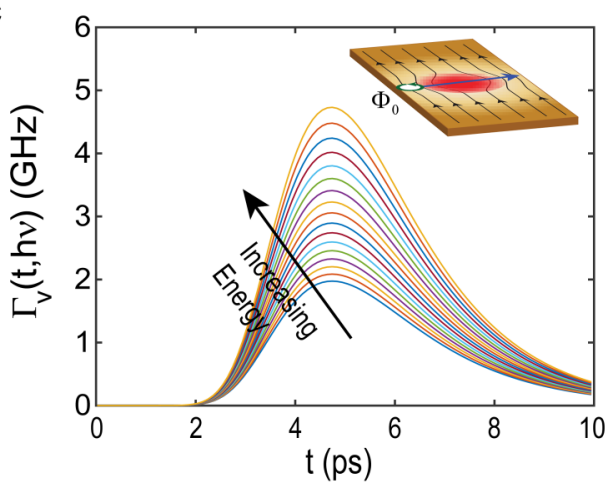

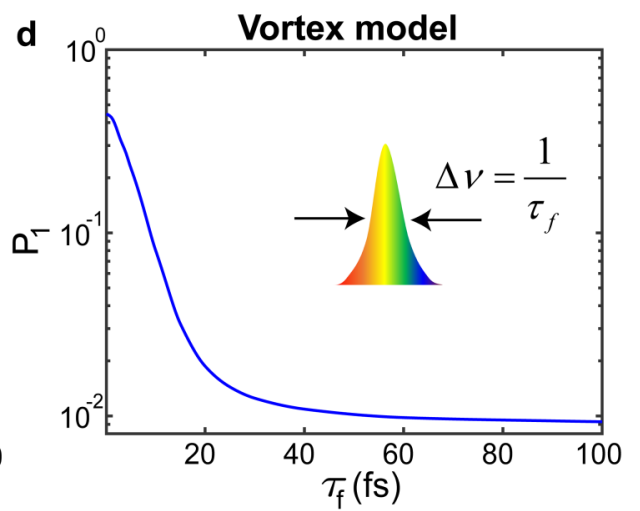

FIG. 10. Energy-dependence and pulse-width dependence of the quantum efficiency. (a) Normalized QP numbers inside the $\xi$-slab, (b) normalized current density at the edge, and (c) vortex crossing rate for different modes of a photon pulse with the central energy of $h v=0.75 \mathrm{eV}$ and a pulse width of $\tau_{f}=100 \mathrm{fs} ; T=0.6 \mathrm{~K}$ and $W=100 \mathrm{~nm}$. The insets illustrate the schematic and the detection criteria for each model. It is seen that the number of QPs and current at the edge are not very sensitive to the small change in the photon energy. However, the single-vortex crossing rate is extremely sensitive as a result of a few percent change in the photon energy. (d) Single-photon quantum efficiency vs the pulse width in the vortex model. The quantum efficiency is considerably increased for the very short pulses. This experimental test can verify the validity of our model. 
up, more changes in QP and current distributions are observed. This causes further suppression of the vortex potential barrier, leading to a higher probability of the vortex crossing. This in turn results in a higher quantum efficiency. Our vortex model by itself can explain both constant efficiency at high energies and an exponential decrease of quantum efficiency at lower energies seen in experiments. ${ }^{59,60}$ Note that the photon absorption efficiency is assumed to be one over the entire spectrum. In practice, the absorption efficiency of a bare nanowire is not very high and does not vary significantly at optical frequencies. However, to increase the absorption efficiency, the detector should be placed inside a high-Q cavity ${ }^{61-65}$ or a low-mode size waveguide ${ }^{8,66-70}$ to enhance the spatial overlap between the optical mode of the incoming photon and the superconducting electrons of the detector.

Until now, we have assumed in our model that the incoming photon is a single-mode photon. However, in practice, the photon has a finite pulse width and the bandwidth of the photon may affect the performance of a detector. The response of the detector to a broadband photon can be used as an experimental test to compare across different detection models and verify our theory. In Figs. 10(a)-10(c), we have compared the detection criteria in different models in response to the different modes of a multi-mode single-photon pulse with a central energy of $h v=0.75 \mathrm{eV}$ and a pulse width of $\tau_{f}=100 \mathrm{fs}$. Number of QPs and current at the edge are the main quantities to define the detection criteria in the hotspot model and the QP model. ${ }^{21}$ As shown in Figs. 10(a) and 10(b), the detector performance is not very sensitive when the photon energy is slightly changed around the central frequency of the photon. However, as shown in Fig. 10(c), a small perturbation in the photon energy can make a considerable change in the single-vortex crossing rate since the rate exponentially changes with the vortex potential energy. Figure 10(d) displays the effect of pulse width on the quantum efficiency in our model. It is seen that there is a remarkable change in the quantum efficiency for ultra-short singlephoton pulses. This effect arises due to the exponential tail of the quantum efficiency and clearly differentiates the proposed vortex model from the existing detection mechanisms. A controlled experiment can verify whether our model is correct or not.

\section{CONCLUSION}

In summary, we have proposed a probabilistic detection criterion in SNSPDs based on a single-vortex moving across the width of the detector. We have shown that, even for a non-vanishing vortex potential barrier, there is significant enhancement in the rate of the vortex crossing after the photon absorption, leading to an increase in the click probability. This non-deterministic process insets a considerable intrinsic timing jitter to the detection event. We have shown the trade-space of the timing jitter, quantum efficiency, and dark counts for different superconducting materials and different nanowire structures. We have presented the quantum efficiency spectrum based on our model, which can predict a pulseshaped dependent quantum efficiency in SNSPDs. This effect is negligible in other proposed models. Our model can predict some observables illustrated in Table I, which can be verified experimentally to confirm or reject our model. It applies specifically to probabilistic (quantum) sources of jitter, and further experiments are
TABLE I. Experimental tests and observables to verify the validity of a detection model.

\begin{tabular}{lc}
\hline \hline Experimental test & Observables \\
\hline $\begin{array}{l}\text { Quantum efficiency } \\
\text { spectrum } \\
\text { Response function }\end{array}$ & Exponential decrease at low energies \\
$\begin{array}{l}\text { Timing jitter vs bias } \\
\text { current } \\
\text { Broadband single } \\
\text { photon }\end{array}$ & $\begin{array}{c}\text { energy and shoulder at threshold } \\
\text { Shoulder at threshold }\end{array}$ \\
\hline \hline
\end{tabular}

needed to distinguish such sources from the dominant geometric jitter.

\section{ACKNOWLEDGMENTS}

We thank Sean Molesky, Joseph Maciejko, Rudro Biswajs, and Bhaskaran Muralidharan for discussions. This work is supported by DARPA DETECT ARO Award No. W911NF-18-1-0074.

\section{APPENDIX: DETECTION MECHANISM FORMALISM}

To find the time-dependent current and QP distributions, we use a modified semi-classical diffusion model, which has been originally proposed by Semenov et al. ${ }^{71}$ and developed by Engel and Schilling. ${ }^{21,33}$

\section{Quasi-particle multiplication}

We assume that the photon energy $(h v)$ is considerably larger than the superconducting bandgap $(\Delta)$, yet not large enough to make a phase transition and form a normal conducting core at the position of the photon absorption. Hence, when the photon is absorbed, a hot electron with a probability density of $C_{e}(\vec{r}, t)$ is created. Since the photon energy is usually orders of magnitude larger than the bandgap, when the hot electron diffuses, it breaks a large number of Cooper pairs $(>100$ in the visible range) to QPs with a distribution density of $C_{q p}(\vec{r}, t)$. This causes the hot electrons to lose their energy, and as a result, the multiplication process slows down with a lifetime of $\tau_{q p}$ due to the electron-phonon interaction, ${ }^{21}$

$$
\begin{gathered}
\frac{\partial C_{e}(\vec{r}, t)}{\partial t}=D_{e} \nabla^{2} C_{e}(\vec{r}, t), \\
\frac{\partial C_{q p}(\vec{r}, t)}{\partial t}=D_{q p} \nabla^{2} C_{q p}(\vec{r}, t)-\frac{C_{q p}(\vec{r}, t)}{\tau_{r}} \\
+\frac{\varsigma h v}{\Delta \tau_{q p}}\left(\frac{n_{s e, 0}-C_{q p}(\vec{r}, t)}{n_{s e, 0}}\right) e^{-t / \tau_{q p}} C_{e}(\vec{r}, t),
\end{gathered}
$$

where $D_{e}, D_{q p}, \tau_{r}$, and $n_{s e, 0}$ are the hot-electron diffusion coefficient, quasi-particle diffusion coefficient, recombination time, and density of superconducting electrons before the photon absorption, 
TABLE II. Material parameters near zero temperature used in simulations.

\begin{tabular}{lcccccccc}
\hline \hline & $\begin{array}{c}\Delta \\
(\mathrm{eV})\end{array}$ & $\begin{array}{c}D_{e} \\
\left(\mathrm{~nm}^{2} / \mathrm{ps}\right)\end{array}$ & $\begin{array}{c}D_{q p} \\
\left(\mathrm{~nm}^{2} / \mathrm{ps}\right)\end{array}$ & $\begin{array}{c}\xi \\
(\mathrm{nm})\end{array}$ & $\begin{array}{c}\lambda \\
(\mathrm{nm})\end{array}$ & $\begin{array}{c}\tau_{r} \\
(\mathrm{ps})\end{array}$ & $\begin{array}{c}\tau_{q p} \\
(\mathrm{ps})\end{array}$ & $\begin{array}{c}\varsigma \\
(\%)\end{array}$ \\
\hline $\mathrm{TaN}$ & 1.3 & 8.2 & 60 & 5.3 & 520 & 1000 & 1.6 & 25 \\
$\mathrm{NbN}$ & 2.3 & 7.1 & 52 & 4.3 & 430 & 1000 & 1.6 & 25 \\
$\mathrm{WSi}$ & 0.53 & 10.3 & 75 & 8 & 1400 & 1000 & 1.6 & 25 \\
\hline
\end{tabular}

respectively. $\varsigma$ is the QP conversion efficiency, which has been assumed constant. We add the term $\left(n_{s e, 0}-C_{q p}(\vec{r}, t)\right) / n_{s e, 0}$ to include the saturation of QP multiplication. We have ignored the electron-phonon and phonon-phonon interactions, which are considerably slower than the electron-electron interactions. ${ }^{21,72}$ The exact solution of the above equation in a general form is not easy to derive. Hence, to find the solution numerically, we have used a finite-difference Crank-Nicolson method. Since the hot electrons diffuse quickly $\left(D_{e} \gg D_{q p}\right)$, to speed-up the simulations, we have used the analytical solution of Eq. (A1) for the case of an infinite $2 \mathrm{D}$ superconductor. ${ }^{21}$ We have assumed a Gaussian distribution for the electron, which is a delta function at $t=0$ and the electron diffuses for $t>0$. A grid size of $\Delta x=\Delta y=1-3 \mathrm{~nm}$ and a time step of $D_{q p} \Delta t / \Delta x^{2}=0.01$ is used in our simulations. The Neumann boundary condition for the sidewalls and zero-flux at the two ends of the nanowire have been considered. The material parameters can be derived from experimental measurements. ${ }^{33,38}$ The parameters that we have used in this work are listed in Table II.

\section{Current redistribution}

The current distribution can be calculated by combining the superconducting phase coherence condition and continuity equation, $^{73}$

$$
\nabla \cdot(\vec{j}(\vec{r}, t))=\nabla \cdot\left(\frac{\hbar}{m} n_{s e}(\vec{r}, t) \nabla \varphi(\vec{r}, t)\right)=0,
$$

where $n_{s e}(\vec{r}, t)=n_{s e 0}-C_{q p}(\vec{r}, t)$ is the density of superconducting electrons after the photon absorption, $\varphi$ is the phase of the superconducting order parameter, and $m$ and $\hbar$ are the electron mass and reduced Planck constant, respectively.

\section{Single-vortex crossing}

Vortices and antivortices are the topological defects in thin superconducting films, which exist even if there is no applied magnetic field. ${ }^{73}$ Vortices are usually nucleated and enter into the nanowire from the edge where the superconducting order parameter is suppressed. The London equation in the presence of a static vortex in a superconducting thin film in the $x y$ plane can be written as ${ }^{73,74}$

$$
\vec{H}(r)+2 \pi \frac{\Lambda}{c} \nabla \times \vec{j}(r)=\hat{z} \Phi_{0} \delta\left(\vec{r}-\vec{r}_{v}\right)
$$

where $\Lambda=2 \lambda^{2} / d$ is the Pearl length, ${ }^{75} \lambda$ is the London penetration depth, $d$ is the film thickness, $\Phi_{0}=h c / 2 e$ is the magnetic flux quantum due to the presence of a single vortex at the position $\vec{r}_{v}$, $\vec{H}$ is the magnetic field, $\vec{j}$ is the current density ignoring the effect of the vortex on the current, and $c$ is the speed of light in vacuum. Since the thickness of the nanowire is significantly smaller than $\lambda$, we have averaged the field and the current in the $z$ direction. For nanoscale SNSPDs $(L \ll \Lambda)$, the first term can be neglected, ${ }^{74}$ and because of the current continuity $(\nabla \cdot \vec{j}=0)$, we can write the current density in the form of a scalar function as $\vec{j}(r)=\nabla \times G(r) \hat{z}$. Thus, Eq. (A4) is reduced to ${ }^{74}$

$$
\nabla^{2} G(r)=-\frac{c \Phi_{0}}{2 \pi \Lambda} \delta\left(\vec{r}-\vec{r}_{v}\right)
$$

which is equivalent to the 2D Poisson's equation for a charged particle. For an infinite superconducting film case, the interaction energy between vortices and antivortices for distances shorter than the Pearl length is logarithmic. This allows Berezinskii-ThoulessKosterlitz (BKT) transition and the formation of vortex-antivortex pairs below the BKT critical temperature. ${ }^{74,76}$ However, for a thin superconductor with a finite width $(-W / 2<x<W / 2)$, the long range interaction between vortices and antivortices is eliminated and single vortices can be found. For a single vortex, Eq. (A5) is reduced to the equation for a charge sandwiched between two parallel grounded plates. The problem is well-known in electrostatics and can be solved using conformal mapping with $z^{\prime}=e^{i \pi z / W}$ transformation and using the image theory,

$$
G(x, y)=\frac{c \Phi_{0}}{8 \pi \Lambda} \ln \frac{\cosh (y \pi / W)+\cos \left(\left(x+x_{v}\right) \pi / W\right)}{\cosh (y \pi / W)-\cos \left(\left(x-x_{v}\right) \pi / W\right)},
$$

where we have assumed that the vortex is placed at $x=x_{v}$ and $y=0$. The phase of the order parameter, $\varphi$, can also be derived from $G$ since the gradient of $\varphi$ is also proportional to the current, ${ }^{28}$

$$
\varphi\left(\vec{r}, \vec{r}_{v}\right)=\tan ^{-1} \frac{\cos \left(\frac{\pi x}{W}\right) \sinh \left(\pi \frac{y-y_{v}}{W}\right)}{\sin \left(\frac{\pi x}{W}\right)-\cosh \left(\pi \frac{y-y_{v}}{W}\right) \sin \left(\frac{\pi x_{v}}{W}\right)} .
$$

The free energy in the presence of a vortex consists of the field energy and the kinetic energy inside the nanowire and the field energy outside. ${ }^{73,74}$ If we assume the vortex core radius is $\xi$ and we neglect the core energy of the vortex, the self-energy of the vortex can be written as ${ }^{20}$

$$
\begin{aligned}
U_{v}^{0}\left(x_{v}\right) & =-\frac{\Phi_{0}}{2 c} G\left(\left|x-x_{v}\right| \rightarrow \xi, 0\right) \\
& =\frac{\Phi_{0}^{2}}{8 \pi^{2} \Lambda} \ln \left(\frac{2 W}{\pi \xi} \cos \left(\frac{\pi x_{v}}{W}\right)\right) .
\end{aligned}
$$

If we include the work done by the bias current on a single vortex due to the Magnus force (dual of the Lorentz force on a magnetic flux), the total energy of a single vortex is expressed $a^{28}$

$$
U_{v}\left(x_{v}\right)=\frac{\Phi_{0}^{2}}{8 \pi^{2} \Lambda} \ln \left(\frac{2 W}{\pi \xi} \cos \left(\frac{\pi x_{v}}{W}\right)\right)-\frac{\Phi_{0}}{c} j_{y}\left(x_{v}\right)\left(x_{v}+\frac{W}{2}\right) .
$$

The Magnus force tries to move the vortex in the direction perpendicular to the direction of the applied bias current, but it 
cannot overcome the self-energy of the vortex if the bias current is not high enough. Increasing the bias current at the edges $j_{y}\left(x_{v}, t\right)$ due to the photon absorption reduces the potential barrier and eases vortex crossing. This barrier finally turns to zero at the vortex critical current, which is

$$
I_{c, v}=\frac{c \Phi_{0}}{4 \pi^{2} \exp (1) \Lambda \xi} W
$$

As seen in Eq. (A7), the phase of the order parameter depends on the position of the vortex, $x_{v}$, and the phase difference at the two ends of a long nanowire $(L \gg W)$ away from the vortex position can be approximated as

$$
\varphi(L / 2)-\varphi(-L / 2)=2 \pi x_{v} / W .
$$

Hence, as the vortex moves across the width of the nanowire, it applies a time-dependent phase difference between the two terminals of the detector. If the vortex crosses from one edge at $x_{v}=-W / 2$ to another edge at $x_{v}=W / 2$, it causes a $2 \pi$ phase-slip at the two ends of the nanowire. This phase evolution generates a voltage pulse, which can be described by the Josephson effect, ${ }^{77}$

$$
V(t)=\frac{\Phi_{0}}{2 \pi c} \frac{d}{d t}(\varphi(L / 2)-\varphi(-L / 2))=\frac{\Phi_{0}}{c W} \frac{d x_{v}}{d t} .
$$

This voltage pulse propagates to the two ends ${ }^{6,78}$ and is dissipated in the presence of the bias current. If the bias current is high enough, the released energy is enough to induce a phase transition in the nanowire from the superconducting state to the normal conducting state. Hence, the current $I_{c, v}$, which makes the vortex tunneling barrier reduce to zero, is also the critical current for phase transition in a thin superconducting nanowire. This critical current is less than the depairing critical current in a bulk superconductor, $I_{c, \text { dep. }}$. 19,21

Even if the applied bias current is below $I_{c, v}$, breaking of the Cooper pairs and redistribution of the bias current due to the photon absorption can also change the potential barrier, ${ }^{21}$

$$
\begin{aligned}
\frac{U_{v}\left(x_{v}, t\right)}{\varepsilon_{0}}= & \frac{\pi}{W} \int_{\frac{\xi-W}{2}}^{x_{v}} \frac{n_{s e}\left(x^{\prime}, t\right)}{n_{s e, 0}} \tan \left(\frac{\pi x^{\prime}}{W}\right) d x^{\prime} \\
& -\frac{2 W}{I_{c, v} \exp (1) \xi} \int_{-\frac{W}{2}}^{x_{v}} \frac{n_{s e}\left(x^{\prime}, t\right)}{n_{s e, 0}} j_{y}\left(x^{\prime}, t\right) d x^{\prime},
\end{aligned}
$$

where $\varepsilon_{0}=\Phi_{0}^{2} / 8 \pi^{2} \Lambda$ is the characteristic vortex energy.

\section{REFERENCES}

${ }^{\mathbf{1}}$ R. H. Hadfield, Nat. Photonics 3, 696 (2009).

${ }^{2}$ G. N. Gol'tsman, O. Okunev, G. Chulkova, A. Lipatov, A. Semenov, K. Smirnov, B. Voronov, A. Dzardanov, C. Williams, and R. Sobolewski, Appl. Phys. Lett. 79, 705 (2001).

${ }^{3}$ C. M. Natarajan, M. G. Tanner, and R. H. Hadfield, Supercond. Sci. Technol. 25, 063001 (2012)

${ }^{4}$ H. Takesue, S. W. Nam, Q. Zhang, R. H. Hadfield, T. Honjo, K. Tamaki, and Y. Yamamoto, Nat. Photonics 1, 343 (2007).
${ }^{\mathbf{5}}$ M. D. Eisaman, J. Fan, A. Migdall, and S. V. Polyakov, Rev. Sci. Instrum. 82, 071101 (2011).

${ }^{6}$ Q.-Y. Zhao, D. Zhu, N. Calandri, A. E. Dane, A. N. McCaughan, F. Bellei, H.-Z. Wang, D. F. Santavicca, and K. K. Berggren, Nat. Photonics 11, 247 (2017).

${ }^{7}$ I. Holzman and Y. Ivry, Adv. Quantum Technol. 2, 1800058 (2019).

${ }^{8}$ F. Marsili, V. B. Verma, J. A. Stern, S. Harrington, A. E. Lita, T. Gerrits, I. Vayshenker, B. Baek, M. D. Shaw, R. P. Mirin, and S. W. Nam, Nat. Photonics 7, 210 (2013).

${ }^{9}$ B. Korzh, Q. Zhao, J. P. Allmaras et al., Nat. Photonics 14, 250-255 (2020).

${ }^{10}$ B. Korzh, Q.-Y. Zhao, S. Frasca, D. Zhu, E. Ramirez, E. Bersin, M. Colangelo, A. Dane, A. Beyer, J. Allmaras et al., in CLEO: QELS_Fundamental Science (Optical Society of America, 2018), pp. FW3F.3.

${ }^{11}$ M. Sidorova, A. Semenov, A. Kuzmin, I. Charaev, S. Doerner, and M. Siegel, IEEE Trans. Appl. Supercond. 28, 2836989 (2018).

${ }^{12}$ W. H. P. Pernice, C. Schuck, O. Minaeva, M. Li, G. N. Goltsman, A. V. Sergienko, and H. X. Tang, Nat. Commun. 3, 1325 (2012).

${ }^{13}$ M. Tarkhov, J. Claudon, J. P. Poizat, A. Korneev, A. Divochiy, O. Minaeva, V. Seleznev, N. Kaurova, B. Voronov, A. V. Semenov, and G. Gol'tsman, Appl. Phys. Lett. 92, 241112 (2008).

${ }^{14}$ C. Schuck, W. H. P. Pernice, and H. X. Tang, Sci. Rep. 3, 1893 (2013).

${ }^{15}$ I. Charaev, A. Semenov, S. Doerner, G. Gomard, K. Ilin, and M. Siegel, Supercond. Sci. Technol. 30, 025016 (2016).

${ }^{16}$ D. Zhu, M. Colangelo, B. A. Korzh, Q.-Y. Zhao, S. Frasca, A. E. Dane, A. E. Velasco, A. D. Beyer, J. P. Allmaras, E. Ramirez et al., Appl. Phys. Lett. 114, 042601 (2019).

${ }^{17}$ M. Sidorova, A. Semenov, H.-W. Hübers, A. Kuzmin, S. Doerner, K. Ilin, M. Siegel, I. Charaev, and D. Vodolazov, Phys. Rev. B 98, 134504 (2018).

${ }^{18}$ K. Epstein, A. M. Goldman, and A. M. Kadin, Phys. Rev. Lett. 47, 534 (1981).

${ }^{19}$ J. Renema, R. Gaudio, Q. Wang, Z. Zhou, A. Gaggero, F. Mattioli, R. Leoni, D. Sahin, M. de Dood, A. Fiore, and M. van Exter, Phys. Rev. Lett. 112, 117604 (2014)

${ }^{20}$ L. N. Bulaevskii, M. J. Graf, and V. G. Kogan, Phys. Rev. B 85, 014505 (2012).

${ }^{21}$ A. Engel and A. Schilling, J. Appl. Phys. 114, 214501 (2013).

${ }^{22}$ A. Engel, J. Renema, K. Il'in, and A. Semenov, Supercond. Sci. Technol. 28, 114003 (2015).

${ }^{23}$ D. Y. Vodolazov, Phys. Rev. Appl. 7, 034014 (2017).

${ }^{24}$ F. Marsili, M. J. Stevens, A. Kozorezov, V. B. Verma, C. Lambert, J. A. Stern, R. D. Horansky, S. Dyer, S. Duff, D. P. Pappas, A. E. Lita, M. D. Shaw, R. P. Mirin, and S. W. Nam, Phys. Rev. B 93, 094518 (2016).

${ }^{25}$ Y. Korneeva, I. Florya, S. Vdovichev, M. Moshkova, N. Simonov, N. Kaurova, A. Korneev, and G. Goltsman, IEEE Trans. Appl. Supercond. 27, 2201504 (2017)

${ }^{\mathbf{2 6}}$ A. Semenov, A. Engel, H.-W. Hübers, K. Il'in, and M. Siegel, Eur. Phys. J. B 47, 495 (2005).

${ }^{27}$ A. N. Zotova and D. Y. Vodolazov, Phys. Rev. B 85, 024509 (2012).

${ }^{28}$ L. N. Bulaevskii, M. J. Graf, C. D. Batista, and V. G. Kogan, Phys. Rev. B 83, 144526 (2011).

${ }^{29}$ D. Y. Vodolazov, Y. P. Korneeva, A. Semenov, A. Korneev, and G. Goltsman, Phys. Rev. B 92, 104503 (2015).

${ }^{30}$ Y. P. Korneeva, D. Y. Vodolazov, A. Semenov, I. Florya, N. Simonov, E. Baeva, A. Korneev, G. Goltsman, and T. Klapwijk, Phys. Rev. Appl. 9, 064037 (2018).

${ }^{31}$ E. Knehr, A. Kuzmin, M. Ziegler, S. Doerner, K. Ilin, M. Siegel, R. Stolz, and H. Schmidt, arXiv:1906.05214 (2019).

${ }^{32}$ J. Allmaras, A. Kozorezov, B. Korzh, K. Berggren, and M. Shaw, Phys. Rev. Appl. 11, 034062 (2019).

${ }^{33}$ A. Engel, J. Lonsky, X. Zhang, and A. Schilling, IEEE Trans. Appl. Supercond. 25, 1 (2015).

${ }^{34}$ M. K. Akhlaghi and A. H. Majedi, IEEE Trans. Appl. Supercond. 19, 361 (2009).

${ }^{35}$ M. K. Akhlaghi, A. H. Majedi, and J. S. Lundeen, Opt. Express 19, 21305 (2011).

${ }^{36}$ S. van Enk, J. Phys. Commun. 1, 045001 (2017).

${ }^{37}$ S. J. van Enk, Phys. Rev. A 96, 033834 (2017). 
${ }^{38}$ H. Bartolf, A. Engel, A. Schilling, K. Il'in, M. Siegel, H.-W. Hübers, and A. Semenov, Phys. Rev. B 81, 024502 (2010).

${ }^{39}$ A. Eftekharian, H. Atikian, M. K. Akhlaghi, A. Jafari Salim, and A. Hamed Majedi, Appl. Phys. Lett. 103, 242601 (2013).

${ }^{40}$ T. Yamashita, S. Miki, K. Makise, W. Qiu, H. Terai, M. Fujiwara, M. Sasaki, and Z. Wang, Appl. Phys. Lett. 99, 161105 (2011).

${ }^{41}$ J. R. Clem and K. K. Berggren, Phys. Rev. B 84, 174510 (2011).

${ }^{42}$ A. Murphy, A. Semenov, A. Korneev, Y. Korneeva, G. Gol'tsman, and A. Bezryadin, Sci. Rep. 5, 10174 (2015).

${ }^{43}$ J. Zhang, W. Słysz, A. Pearlman, A. Verevkin, R. Sobolewski, O. Okunev, G. Chulkova, and G. Gol'tsman, Phys. Rev. B 67, 132508 (2003).

${ }^{44}$ L. P. Yang, H. X. Tang, and Z. Jacob, Phys. Rev. A 97, 013833 (2018).

${ }^{45}$ L. P. Yang and Z. Jacob, Opt. Express 27, 10482 (2019).

${ }^{46} \mathrm{H}$. Wu, C. Gu, Y. Cheng, and X. Hu, Appl. Phys. Lett. 111, 062603 (2017).

${ }^{47}$ N. Calandri, Q.-Y. Zhao, D. Zhu, A. Dane, and K. K. Berggren, Appl. Phys. Lett. 109, 152601 (2016).

${ }^{48}$ J. A. O'Connor, M. G. Tanner, C. M. Natarajan, G. S. Buller, R. J. Warburton, S. Miki, Z. Wang, S. W. Nam, and R. H. Hadfield, Appl. Phys. Lett. 98, 201116 (2011).

${ }^{49}$ Y. Cheng, C. Gu, and X. Hu, Appl. Phys. Lett. 111, 062604 (2017).

${ }^{50}$ D. Y. Vodolazov, Phys. Rev. Appl. 11, 014016 (2019).

${ }^{51}$ A. Kuzmin, S. Doerner, S. Wuensch, K. Ilin, M. Siegel, M. Sidorova, and A. Semenov, IEEE Trans. Appl. Supercond. 29, 2201105 (2019).

${ }^{52}$ R. Cheng, C.-L. Zou, X. Guo, et al., Nat. Commun. 10, 4104 (2019).

${ }^{53}$ J. J. Renema, Q. Wang, R. Gaudio, I. Komen, K. Op't Hoog, D. Sahin, A. Schilling, M. van Exter, A. Fiore, A. Engel et al., Nano Lett. 15, 4541 (2015).

${ }^{54}$ D. F. Santavicca, B. Noble, C. Kilgore, G. A. Wurtz, M. Colangelo, D. Zhu, and K. Berggren, IEEE Trans. Appl. Supercond. 29, 2200504 (2019).

${ }^{55}$ J. Renema, G. Frucci, Z. Zhou, F. Mattioli, A. Gaggero, R. Leoni, M. J. de Dood, A. Fiore, and M. P. van Exter, Phys. Rev. B 87, 174526 (2013).

${ }^{56}$ M. Caloz, B. Korzh, N. Timoney, M. Weiss, S. Gariglio, R. J. Warburton, C. Schönenberger, J. Renema, H. Zbinden, and F. Bussières, Appl. Phys. Lett. 110, 083106 (2017).

${ }^{57}$ M. Sidorova, A. Semenov, H.-W. Hübers, I. Charaev, A. Kuzmin, S. Doerner, and M. Siegel, Phys. Rev. B 96, 184504 (2017).
${ }^{58}$ M. Caloz, M. Perrenoud, C. Autebert, B. Korzh, M. Weiss, C. Schönenberger, R. J. Warburton, H. Zbinden, and F. Bussières, Appl. Phys. Lett. 112, 061103 (2018).

${ }^{\mathbf{5 9}}$ A. D. Semenov, P. Haas, H.-W. Hübers, K. Ilin, M. Siegel, A. Kirste, T. Schurig, and A. Engel, Physica C 468, 627 (2008).

${ }^{60}$ M. Hofherr, D. Rall, K. Ilin, M. Siegel, A. Semenov, H.-W. Hübers, and N. Gippius, J. Appl. Phys. 108, 014507 (2010).

${ }^{61}$ K. M. Rosfjord, J. K. W. Yang, E. A. Dauler, A. J. Kerman, V. Anant, B. M. Voronov, G. N. Gol'tsman, and K. K. Berggren, Opt. Express 14, 527 (2006).

${ }^{62}$ A. Vetter, S. Ferrari, P. Rath, R. Alaee, O. Kahl, V. Kovalyuk, S. Diewald, G. N. Goltsman, A. Korneev, C. Rockstuhl, and W. H. P. Pernice, Nano Lett. 16, 7085 (2016).

${ }^{63}$ J. Münzberg, A. Vetter, F. Beutel, W. Hartmann, S. Ferrari, W. H. Pernice, and C. Rockstuhl, Optica 5, 658 (2018).

${ }^{64}$ K. Erotokritou, R. Heath, G. Taylor, C. Tian, A. Banerjee, A. Casaburi, C. Natarajan, S. Miki, H. Terai, and R. H. Hadfield, Supercond. Sci. Technol. 31, $125012(2018)$

${ }^{65}$ Y. Yun, A. Vetter, R. Stegmueller, S. Ferrari, W. H. Pernice, C. Rockstuhl, and C. Lee, Phys. Rev. Appl. 13, 014061 (2020).

${ }^{66}$ S. Ferrari, C. Schuck, and W. Pernice, Nanophotonics 7, 1725 (2018).

${ }^{67}$ F. Najafi, J. Mower, N. C. Harris, F. Bellei, A. Dane, C. Lee, X. Hu, P. Kharel, F. Marsili, S. Assefa, K. K. Berggren, and D. Englund, Nat. Commun. 6, 5873 (2015).

${ }^{68}$ S. Jahani and Z. Jacob, Optica 1, 96 (2014).

${ }^{69}$ S. Jahani, S. Kim, J. Atkinson, J. C. Wirth, F. Kalhor, A. Al Noman, W. D. Newman, P. Shekhar, K. Han, V. Van, R. G. DeCorby, L. Chrostowski, M. Qi, and Z. Jacob, Nat. Commun. 9, 1893 (2018).

${ }^{70}$ M. K. Akhlaghi, E. Schelew, and J. F. Young, Nat. Commun. 6, 8233 (2015).

${ }^{71}$ A. D. Semenov, G. N. Gol'tsman, and A. A. Korneev, Physica C 351, 349 (2001).

${ }^{72}$ Y. P. Gousev, G. Gol'Tsman, A. Semenov, E. Gershenzon, R. Nebosis, M. Heusinger, and K. Renk, J. Appl. Phys. 75, 3695 (1994).

${ }^{73} \mathrm{M}$. Tinkham, Introduction to Superconductivity (Courier Corporation, 1996).

${ }^{74}$ V. G. Kogan, Phys. Rev. B 75, 064514 (2007).

${ }^{75}$ J. Pearl, Appl. Phys. Lett. 5, 65 (1964).

${ }^{76}$ A. M. Kadin, M. Leung, and A. D. Smith, Phys. Rev. Lett. 65, 3193 (1990).

77. R. Clem, J. Low Temp. Phys. 42, 363 (1981).

${ }^{78}$ D. F. Santavicca, J. K. Adams, L. E. Grant, A. N. McCaughan, and K. K. Berggren, J. Appl. Phys. 119, 234302 (2016). 